\title{
Genotyping of Human Papillomavirus in Cervical Liquid- Based Cytology Specimens
}

\section{ART ICLE INF O}

\section{Article Type}

Original research

\section{Authors}

Rezaei $S .{ }^{1} M S C$,

Saadatniya G.* PhD,

Salehian $\mathrm{P}^{2} M D$

\section{How to cite this article}

Rezaei S, Saadatniya G, Salehian P.

Genotyping of Human Papillom-

avirus in Cervical Liquid-Based

Cytology Specimens. Sarem Jo-

urnal of Reproductive Medicine.

2017;1(4):165-169.

\section{A B S T R A C T}

Aims Human papillomavirus (HPV) is the main cause of cervical cancer and the second cause of cancer mortality among women. Molecular epidemiologic evidence clearly demonstrated that certain types of HPV play a major role in the development of cervical cancer. The objective of this study was to genotype the human papillomavirus in cervical liquid-based cytology specimens.

Materials \& Methods This descriptive cross-sectional study was conducted on 42 liquidbased cytology specimens from women (25-50 years old) referred to the laboratory of Sarem women's hospital during one year from 2013 to 2014. Those HPV infections were confirmed by pathological and molecular methods. Polymerase chain reaction (PCR) and hybridization methods were used to determine the genotypes.

Findings Out of the 42 tested samples, 27 cases (64\%) belonged to HPV high-risk group that included the types of 16, 18, 31, 33, 56, 58 and 66. Among those 27 samples, 14 (52\%) had a combination of infections and $70.4 \%$ of the samples showed infection with types 16 and 18 . Conclusion Various types of HPV can be detected in liquid-based cytology specimens and the most common type is HPV16. Simultaneous infections with multiple types of HPV are common in liquid-based cytology specimens of positive women.

Keywords Genotyping; Human Papillomavirus; PCR; Hybridization

\section{I T A T I O N L I N K S}

[1] Chapter 2: The burden of HPV-related cancers [2] Human papillomavirus types 16 and 18 vaccine (recombinant, AS04 adjuvanted, adsorbed) [3] Development and implementation of papillomavirus prophylactic vaccines [4] Human papillomavirus (HPV) and cervical cancer [5] Human papillomavirus and cervical cancer [6] Vaccination against human papillomavirus infection: A new paradigm in cervical cancer control [7] Short report: Influence of HPV type on prognosis in patients diagnosed with invasive cervical cancer [8] Anal cytological abnormalities and anal HPV infection in men with centers for disease control group IV HIV disease [9] Distinct manifestations of Human papilloma viruses in the vagina [10] Determinants of squamous intraepithelial lesions (SIL) on Pap smear: The role of HPV infection and of HIV-1-induced immunosuppression. DIANAIDS collaborative study group [11] Determinants for genital human papilloma virus (HPV) infection in 1000 randomly chosen young Danish with normal pap smear [12] Quadrivalent Human Papillomavirus (Types 6, 11, 16, 18) Recombinant Vaccine (Gardasil): A review of its use in the prevention of premalignant genital lesions, genital cancer and genital warts in women [13] Oncogenic types of human papillomavirus by Multiplex PCR in cervical cancer lesions in the north west of Iran [14] Classification of papillomaviruses [15] Epidemiologic classification of human papillomavirustypes associated with cervical cancer [16] Cytological early detection of cervical carcinoma: Possibilities and limitations, analysis of failures [17] Detection and typing of human papilloma virus DNA in cervical cancer with In situ hybridization method [18] Type-specific HPV prevalence in cervical cancer and high-grade lesions in Latin America and the Caribbean: Systematic review and metaanalysis [19] Prevalence of human papillomavirus (HPV) genotypes and multiple infections in routine cervical cancer screening in a Spanish regional population [20] Developing an HPV vaccine to prevent cervical cancer and genital wart. Vaccine [21] Comparison of the immunogenicity and safety of Cervarix and Gardasil human papillomavirus (HPV) cervical cancer vaccines in healthy women aged 18-45 years [22] Effect of human papillomavirus genotype on severity and prognosis of cervical intraepithelial neoplasia [23] Human Papillomavirus Genotyps in cervical cancer in Yazd [24] Reviewing the Prevalence of Human Papillomavirus (HPV) in Married Women Aged 18-60 Years with Normal Pap Smear and Referring to Gynecology Clinics in Hospitals Affiliated to Isfahan University of Medical Sciences, Iran [25] Frequency of Dysplastic and Cancerous Pap smear and Genotyping of Human Papillomavirus by DNA Probetechniques in Kerman, Iran [26] Human papillomavirus infection prevalence in women referred to health clinic of Shahid Beheshti university of medical sciences, Tehran, Iran

\section{Article History}

Received: April 29, 2016

Accepted:October 11,2016

ePublished: November 15, 2017 


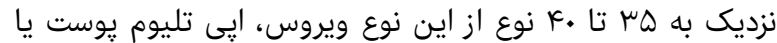

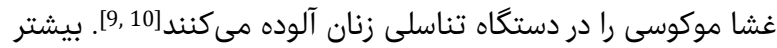

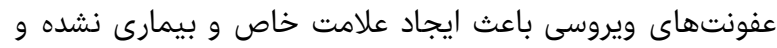

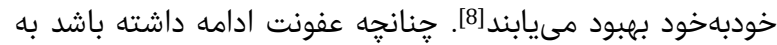

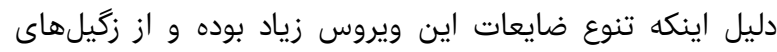

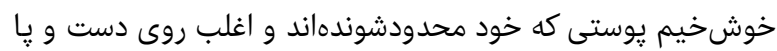

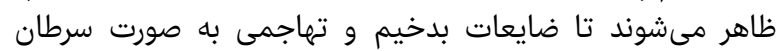

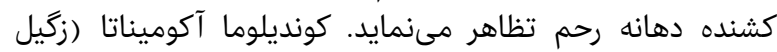

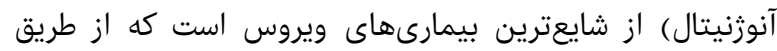
تماس جنسى منتقل مى شود [18-11].

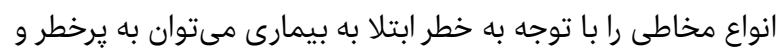

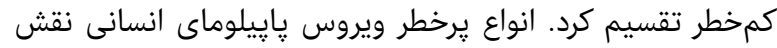

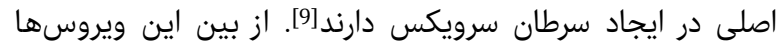

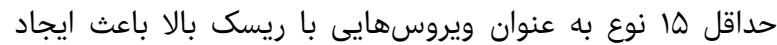

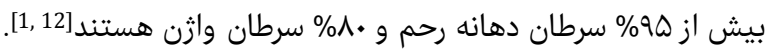

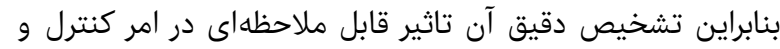

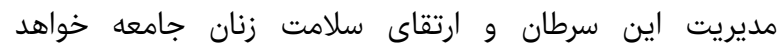

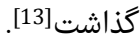

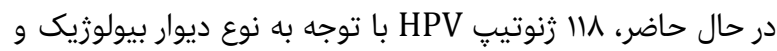

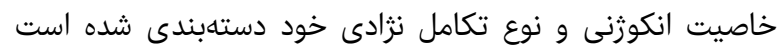

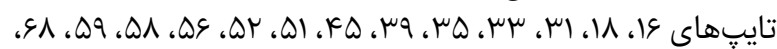

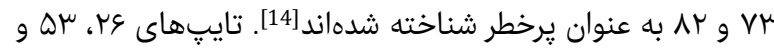

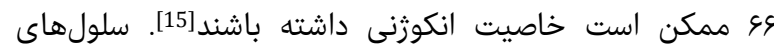

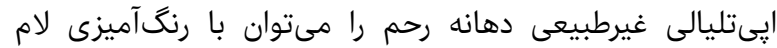

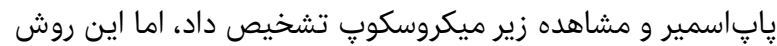

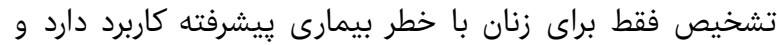

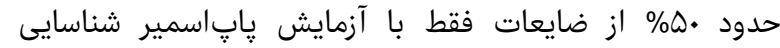

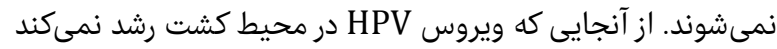

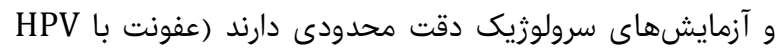

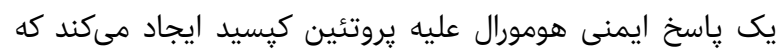

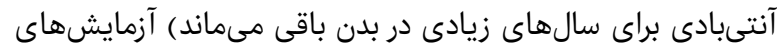

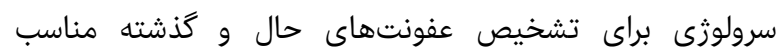

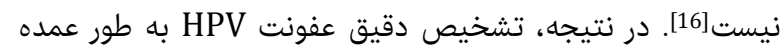

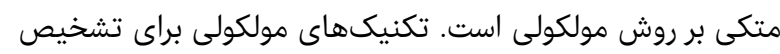

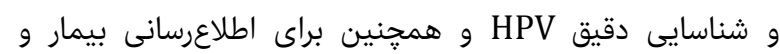

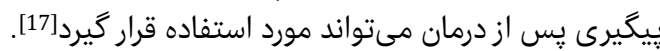

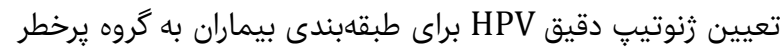

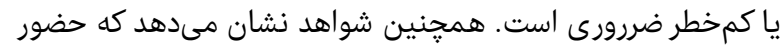

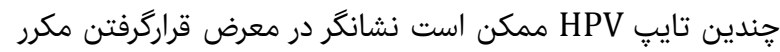

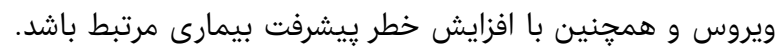

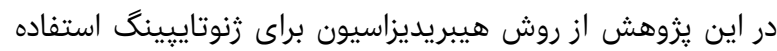

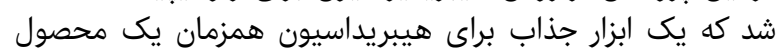

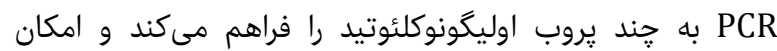

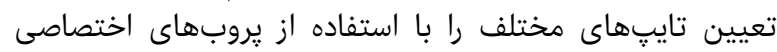

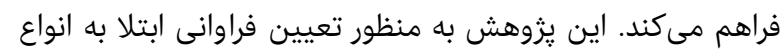

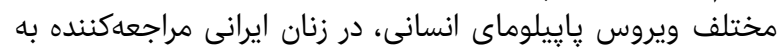

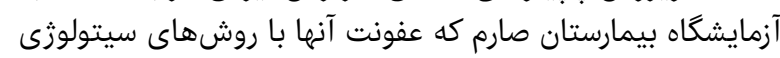

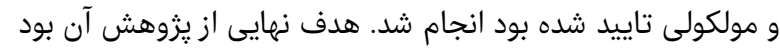

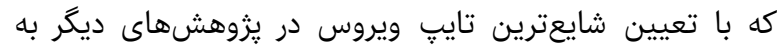
طراحى واكسن نوتركيب عليه آن اقدام شود.
تعيين رنوتيڤهاى ويروس پِإيلوماى انسانى

أو أمميرا رضايى و همكاران در نمونههاى سيتولوثى مايع دهانه (HPV) رحم - سال rqسا بيمارستان فوق تخصصى صائولوري مايع دمان

MSc سميرا رضايى سولى يخوهشكده سلولى- مولكولى و سلولهاى بنى بنيادى صارم، بيمارستان فوق تخصصى صارم، تهران، ايران

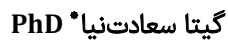

يزوهشكده زيستفناورى، سازمان يُوهشهانهاى علمى و صنعتى ايران، تهران، ايران

MD ييروز صالحيان مركز تحقيقات بارورى و نابارورى صارم، بيمارستان فوق تخصصى صارم، تهران، ايران

جكيده اهذاف: ويروس پإييلوماى انسانى عامل اصلى سرطان دهانه رحم است و دومين

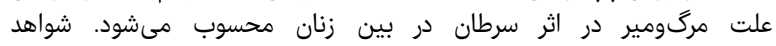

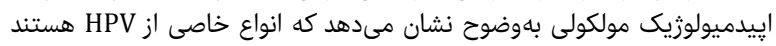

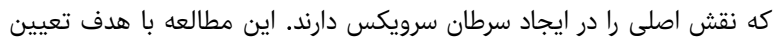

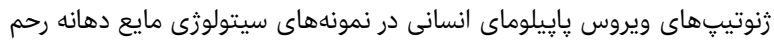

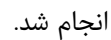

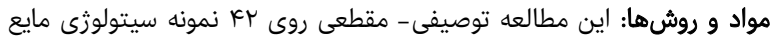

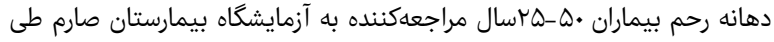

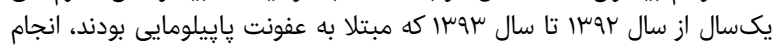

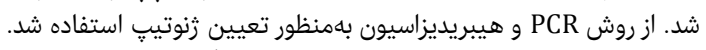

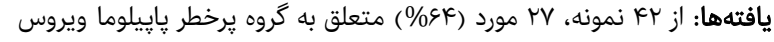

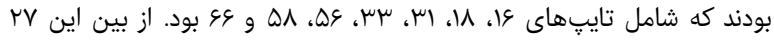

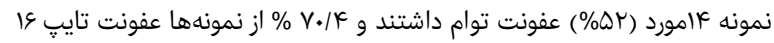

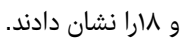

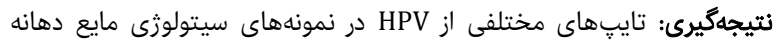

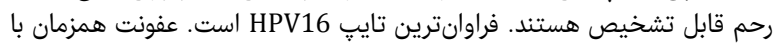

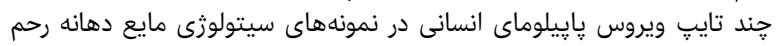

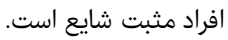
كليدواثهها: زنوتاييينكً، ويروس يإيبيلوماى انسانى، PCR، هيبريديزاسيون

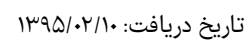

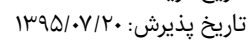

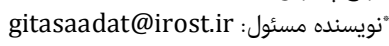

مقدمه مقط

سرطان دهانه رحم دومين سرطان شايع در زنان، با بروز سالانه

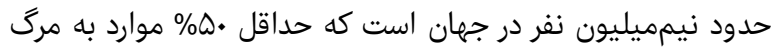

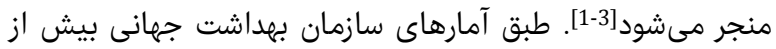

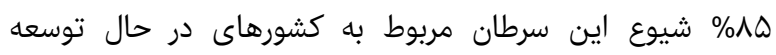

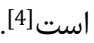

ويروس يإييلوماى انسانى (HPV) به عنوان يكى از مهمترين

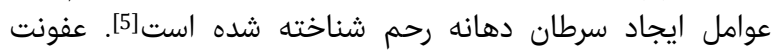

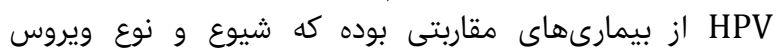

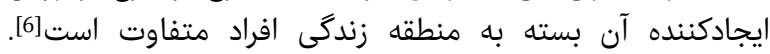

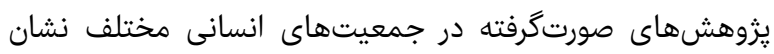

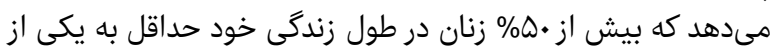

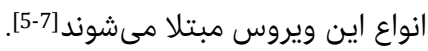

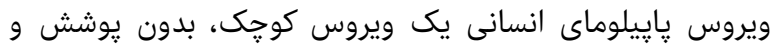

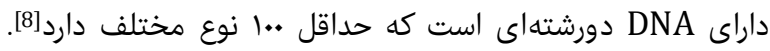




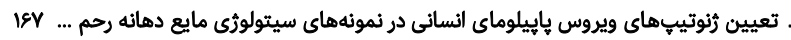

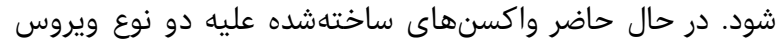

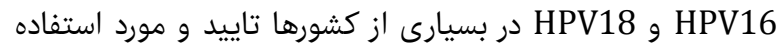
قرار گرفته است $[20,21]$

يثروهشهاى اييدميولوزيك بين المللى نشان مى ترهند كه HPV16

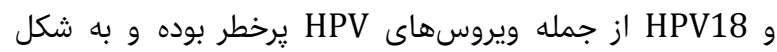

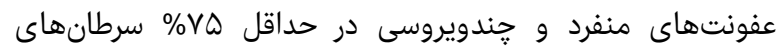

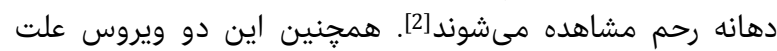

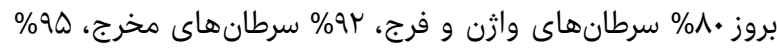

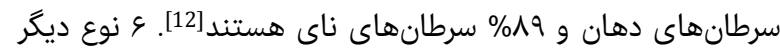

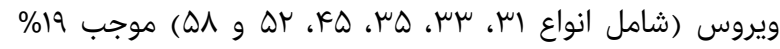

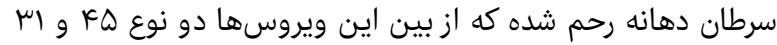

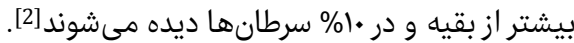

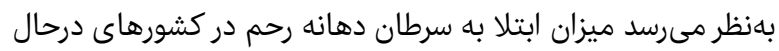

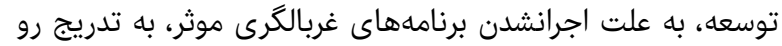

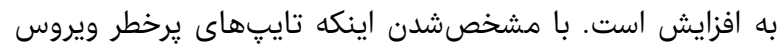

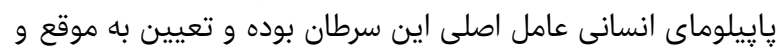

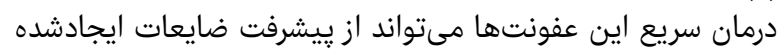

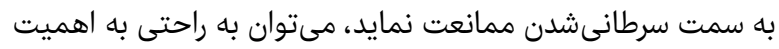

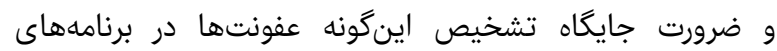

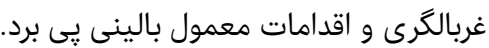

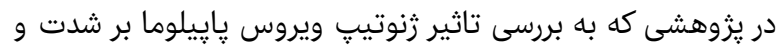

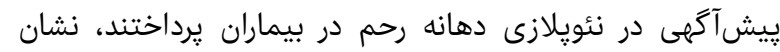

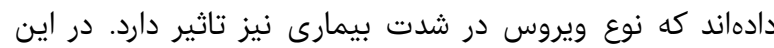

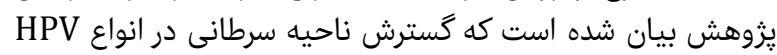

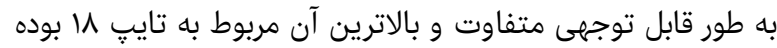

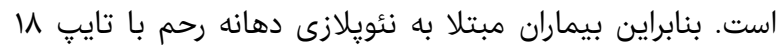

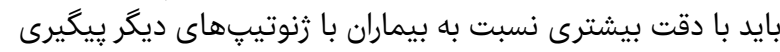

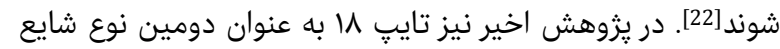

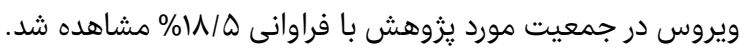

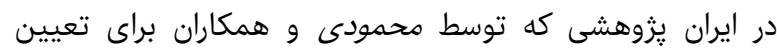

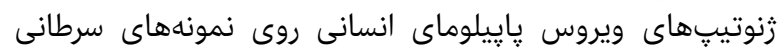

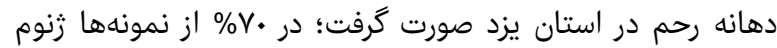

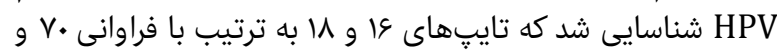

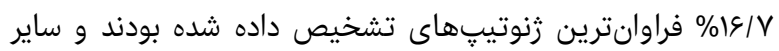

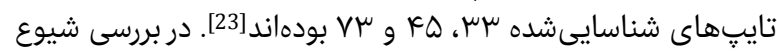

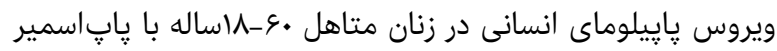

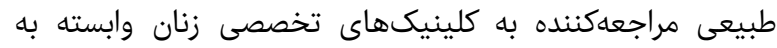

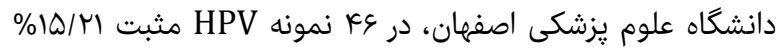

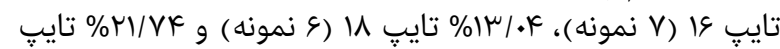

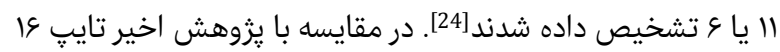
داراى شيوع بسيار كمترى بوده است. داده شئ.

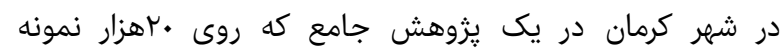

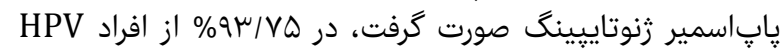

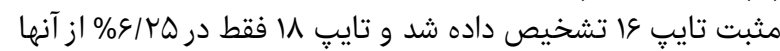

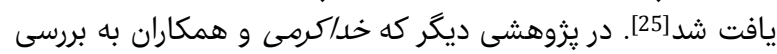

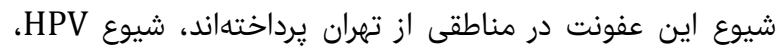

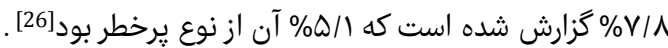

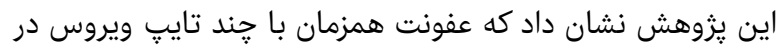

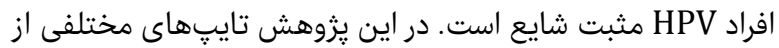

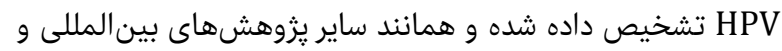

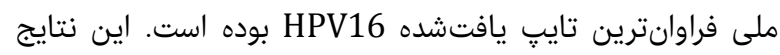

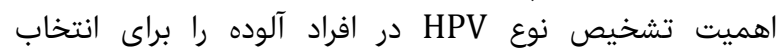

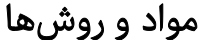

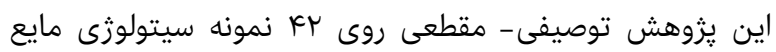

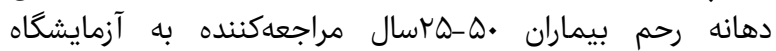

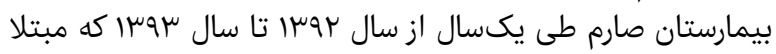

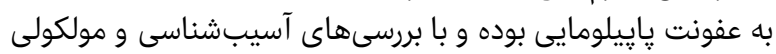

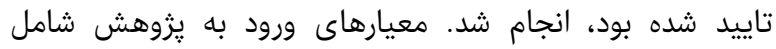

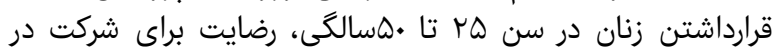

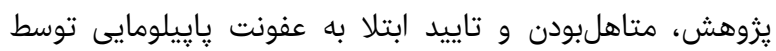

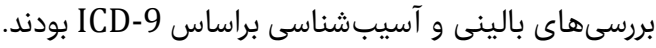

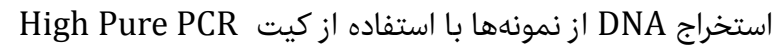
(Roche) Template Preparation kit HPV type 3.5 LCD Array زنوتاييينگ با استفاده از كيت مثبت HPV روى تمامى نمونههاى (Chipron GmbH)

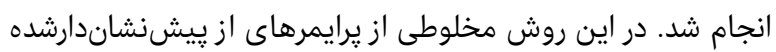

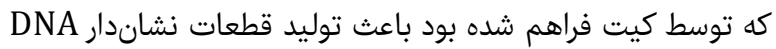

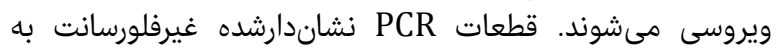

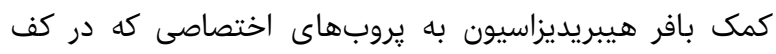

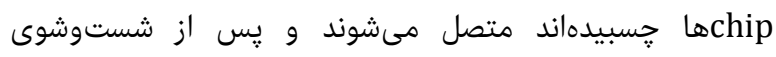

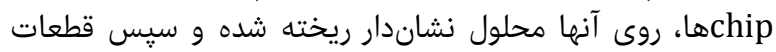

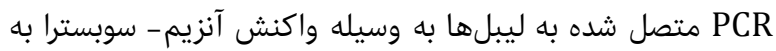

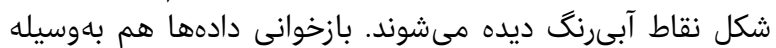

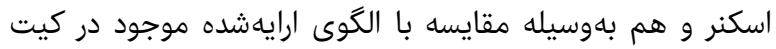
انجام مى اشود. در اين روش دو جفت يرايمر براى PCR فراهم شده بود، يك جفت

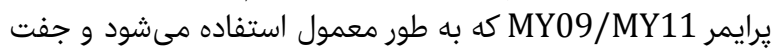

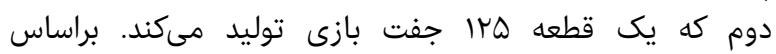

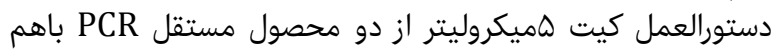

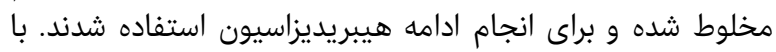

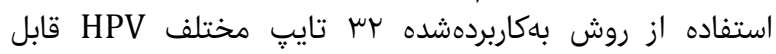

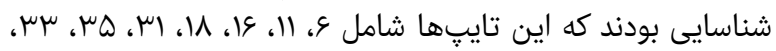

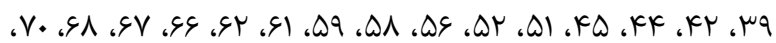
.

\section{يافتهها}

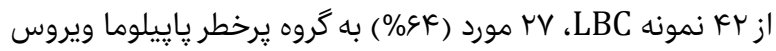

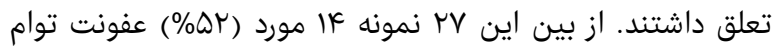

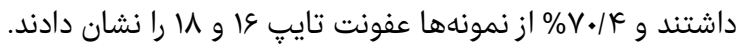

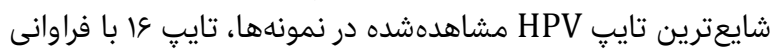

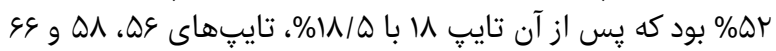

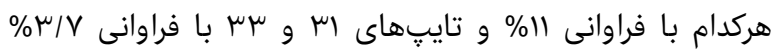

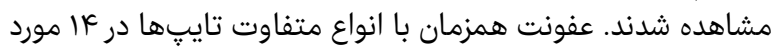

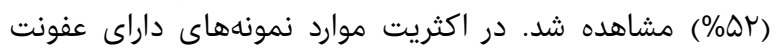

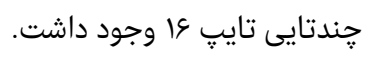

\section{بحث}

تعيين انواع تايڤهاى HPV كه موجب افزايش خطر ابتلا به إنهائ

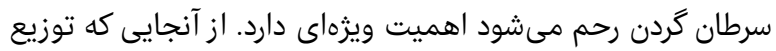

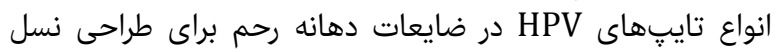

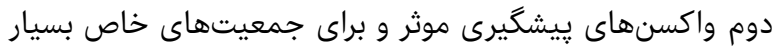

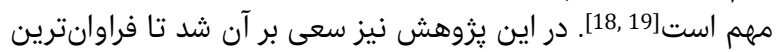

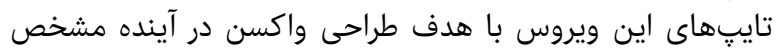


centers for disease control group IV HIV disease. Genitourin Med. 1997;73(3):174-80.

9- Sugase M, Matsukura T. Distinct manifestations of Human papilloma viruses in the vagina. Int J Cancer. 1997;72(3):412-5.

10- Rezza G, Giuliani M, Branca M, Benedetto A, Migliore G, Garbuglia AR, et al. Determinants of squamous intraepithelial lesions (SIL) on Pap smear: The role of HPV infection and of HIV-1-induced immunosuppression. DIANAIDS collaborative study group. Eur J Epidemiol. 1997,13(8):937-43.

11- Kjaer SK, Van Den Brule AJ, Bock JE, Poll PA, Engholm G, Sherman ME, et al. Determinants for genital human papilloma virus (HPV) infection in 1000 randomly chosen young Danish with normal pap smear. Cancer Epidemiol Biomarkers Prev. 1997;6(10):799-805.

12- McCormack PL, Joura EA. Quadrivalent Human Papillomavirus (Types 6, 11, 16, 18) Recombinant Vaccine (Gardasil): A review of its use in the prevention of premalignant genital lesions, genital cancer and genital warts in women. Drugs. 2010;70(18):2449-74.

13- Jabbarpour BM, Esmaeili M, Dastranj A. Oncogenic types of human papillomavirus by Multiplex PCR in cervical cancer lesions in the north west of Iran. Iran J Infect Dis. 2008;13(41):29-34. [Persian]

14- de Villiers EM, Fauquet C, Broker TR, Bernard HU, zur Hausen H. Classification of papillomaviruses. Virology. 2004;324(1):17-27.

15- Muñoz N, Bosch FX, de Sanjosé S, Herrero R, Castellsagué X, Shah KV, et al. Epidemiologic classification of human papillomavirustypes associated with cervical cancer. N Engl J Med. 2003;348(6):518-27.

16- Mobius G. Cytological early detection of cervical carcinoma: Possibilities and limitations, analysis of failures. J Cancer Res Clin Oncol. 1993;119(9):513-21.

17- Nikan M, Garshasbi A, Jalali MR, Gilani M, Faghihzadeh S. Detection and typing of human papilloma virus DNA in cervical cancer with In situ hybridization method. J Reprod Infertil. 2000;1(3):18-22.

18- Ciapponi A, Bardach A, Glujovsky D, Gibbons L, Picconi MA. Type-specific HPV prevalence in cervical cancer and high-grade lesions in Latin America and the Caribbean: Systematic review and meta-analysis. PLoS One. 2011;6(10):e25493.

19- de Mendez MT. Prevalence of human papillomavirus (HPV) genotypes and multiple infections in routine cervical cancer screening in a Spanish regional population. SOJ Microbiol Infect Dis. 2013;1(1):1-6.

20- Bryan JT. Developing an HPV vaccine to prevent cervical cancer and genital wart. Vaccine. 2007;25(16):3001-6.

21- Einstein MH, Baron M, Levin MJ, Chatterjee A Edwards RP, Zepp F, et al. Comparison of the immunogenicity and safety of Cervarix and Gardasil human papillomavirus (HPV) cervical cancer vaccines in healthy women aged 18-45 years. Hum Vaccin. 2009;5(10):705-19.

22- $\mathrm{Ku} \mathrm{CH}$, Lee $\mathrm{SH}$, Lee SP. Effect of human papillomavirus genotype on severity and prognosis of cervical intraepithelial neoplasia. Obstet Gynecol Sci. 2014;57(1):37-43.

23- Mahmoudi MM, Hamkar R, Akhavan-Tafti M, Eslamifar A, Adibi L, Sadrabadi AA, et al. Human Papillomavirus Genotyps in cervical cancer in Yazd. Iran J Infect Dis. 2008;12(37):19-24. [Persian]

24- Allameh T, Moghim S, Farahbod F. Reviewing the Prevalence of Human Papillomavirus (HPV) in Married

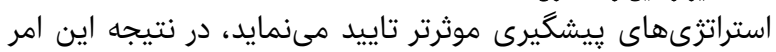

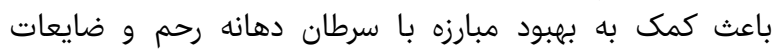

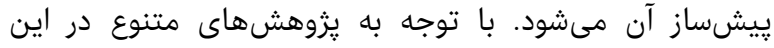

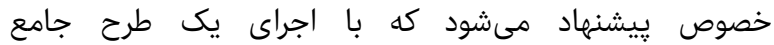

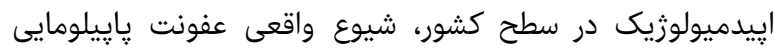

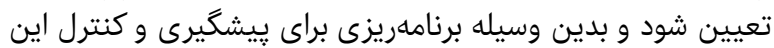

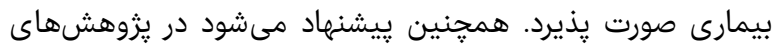

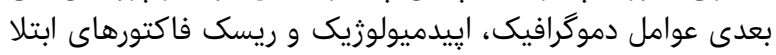
نيز بررسى شود.

نتيجهگيرى
تاييهاى مختلفى از HPV تشخيص داده شداد شد و فراوانترين تايب

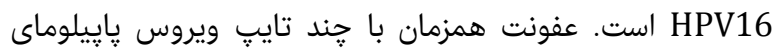
انسانى در نمونههاى سيتولوزى مايع دهانه رحم افراد مثبت ايت شايع يرون يايلوماي

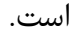

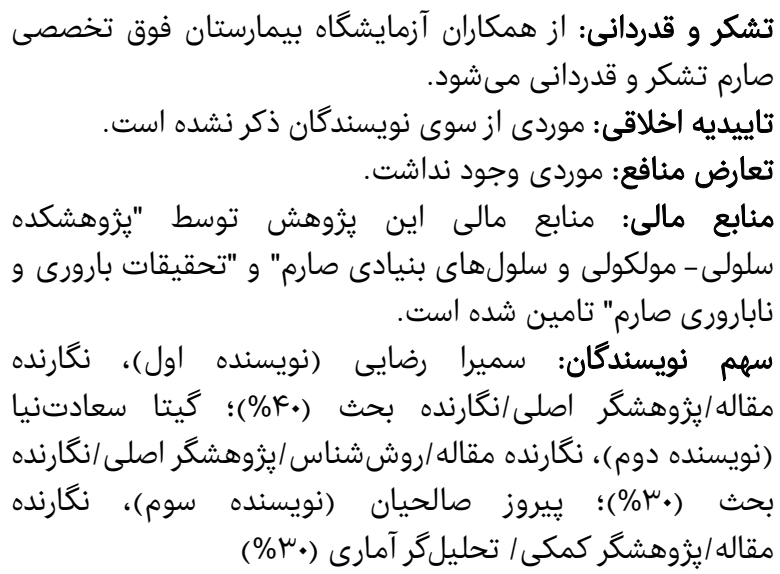
منابع 1- Parkin DM, Bray F. Chapter 2: The burden of HPVrelated cancers. Vaccine, 2006;24(3: S3):11-25.

2- Keam SJ, Harper DM. Human papillomavirus types 16 and 18 vaccine (recombinant, AS04 adjuvanted, adsorbed) [Cervarix]. Drugs. 2008;68(3):359-72.

3- Frazer IH. Development and implementation of papillomavirus prophylactic vaccines. J Immunol. 2014;192(9):4007-11.

4- Human papillomavirus (HPV) and cervical cancer [internet]. World Health Organization: Comprehensive cervical cancer control: A guide to essential practice. 2014. [updated 2016 Jun; cited 2015 Apr]. Available from: http://www.who.int/mediacentre/factsheets/fs380/en. 5- Schiffman M, Castle PE, Jeronimo J, Rodriguez AC, Wacholder S. Human papillomavirus and cervical cancer. Lancet. 2007;370(9590):890-907.

6- Franco EL, Harper DM. Vaccination against human papillomavirus infection: A new paradigm in cervical cancer control. Vaccine. 2005;23(17-18):2388-94.

7- Cuschieri K, Brewster D, Graham C, Nicoll S, Williams A, Murray G, et al. Short report: Influence of HPV type on prognosis in patients diagnosed with invasive cervical cancer. Int J Cancer. 2014;135(11):2721-6.

8- Palefsky JM, Holly EA, Ralston ML, Arthur SP, Hogeboom CJ, Darragh TM. Anal cytological abnormalities and anal HPV infection in men with 
تعيين رنوتييهاى ويروس يإييلوماى انسانى در نمونهاى سيتولوزى مايع دهانه رحم ... Papillomavirus by DNA Probetechniques in Kerman, Iran. J Kerman Univ Med Sci. 2013;20(5):450-9.

26- Khodakarami N, Hosseini SJ, Yavari P, Farzaneh F, Etemad K, Salehpour S, et al. Human papillomavirus infection prevalence in women referred to health clinic of Shahid Beheshti university of medical sciences, Tehran, Iran. Iran J Epidemiol. 2012;7(4):35-42.
Women Aged 18-60 Years with Normal Pap Smear and Referring to Gynecology Clinics in Hospitals Affiliated to Isfahan University of Medical Sciences, Iran. J Isfahan Med Sch. 2012;29(163):1-8.

25- Monsefi N, Dabiri SH, Abbaszadeh M, Safizadeh H, Fotouhi AR, Amirpour RS, et al. Frequency of Dysplastic and Cancerous Pap smear and Genotyping of Human 